\title{
Gasto farmacéutico en medicación antirretroviral y posibilidades de optimización
}

\author{
C. TORNERO ESTÉBANEZ, A. CUENCA SORIA ${ }^{1}$, A. NOLASCO BONMATÍ ${ }^{2}$, \\ E. SOLER COMPANY ${ }^{1}$, S. RULL SEGURA

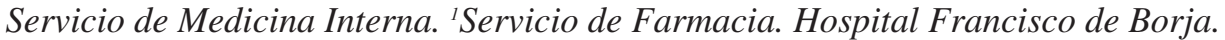 \\ Gandia. Valencia. ${ }^{2}$ Departamento de Enfermería Comunitaria, Medicina Preventiva y \\ Salud Pública e Historia de la Ciencia. Universidad de Alicante
}

\begin{abstract}
ANTIRETROVIRAL PHARMACEUTICAL EXPENDITURE AND POSSIBILITIES FOR OPTIMIZATION
\end{abstract}

\section{RESUMEN}

Introducción: La falta de adherencia, las resistencias y las fases no avanzadas de la enfermedad, hacen que no todos los pacientes con infección por el VIH se beneficien de la demostrada eficacia de las combinaciones de fármacos antirretrovirales, lo que supone una proporción importante del elevado gasto que generan. Nos planteamos analizar, mediante estudio prospectivo de seguimiento de cohortes, como se distribuye el gasto farmacéutico en medicación antirretroviral en un hospital comarcal y las posibilidades de mejorar su eficiencia.

Material y métodos: Se incluyeron 128 pacientes con infección por $\mathrm{VIH}$ atendidos durante 12 semanas consecutivas (septiembre a noviembre del 2004). Se analizaron las características de los seguimientos previos, y, según la evolución en las semanas siguientes y el resultado del tratamiento pautado, se clasificaron los pacientes en grupos: grupo 1 tratamiento efectivo, grupo 2 tratamiento no efectivo y grupo 3 abandonos de tratamiento.

Con la historia de prescripción, corroborada con la recogida de medicación y los costos de adquisición (programa informático FARMASYS $^{\circledast}$ ), se calculó el gasto en medicación antirretroviral generado en cada uno de los 108 pacientes que recibieron TARGA. El análisis estadístico se realizó mediante SPSS 11,5 utilizando como criterio de significación estadística una $\mathrm{p}<0,05$.

Resultados: El gasto total generado en los 108 pacientes incluidos durante el periodo de estudio que recibieron TARGA fue de $232.366 €$, lo que supone una media de $2.151 €$ por paciente y $19.363 €$ por día de consulta. La distribución de los pacientes y del gasto generado por los grupos antes definidos es la siguiente: grupo 1: 79 pacientes $(80,6 \%)$; grupo 2: 11 pacientes $(10,6 \%)$; grupo 3: 18 pacientes $(8,8 \%)$. Entre los pacientes del grupo 1 , un $15,2 \%$ del gasto en terapia antirretroviral se distribuyó a pacientes que la habían iniciado con un recuento de CD4 de más de 350 células $/ \mathrm{ml}$, un $45 \%$ a pacientes que se encontraban en el momento actual con más de 350 células $/ \mathrm{ml}$ y un $25,9 \%$ a pacientes con recuentos de más de 500 células $/ \mathrm{ml}$. El antecedente de abandonos previos de la medicación se comportó como predictor de nuevos abandonos y fracasos terapéuticos.

Comentario: El gasto generado por la medicación antirretoviral es elevado aunque en un $19,4 \%$ se administra a pacientes que no se benefician de él por falta de eficacia o abandonos, y en otro 40 a $60 \%$ podría plantearse su necesidad dada la buena situación inmunológica de los enfermos.

PALABRAS CLAVE: Medicación antirretroviral. Gasto farmacéutico.

\section{ABSTRACT}

Introduction: A lack of treatment compliance, resistances and nonadvanced stages of the disease are the reasons why not all HIV-infected patients benefit from the demonstrated efficacy of antiretroviral drug combinations - with the consequent important increase in costs. A prospective cohort follow-up study is conducted to analyze the distribution of antiretroviral pharmaceutical expenditure in a district hospital, and the possibilities for improving its efficiency.

Material and methods: The study comprised 128 HIV-infected patients seen for 12 consecutive weeks (September to November, 2004). The characteristics of previous follow-up were analyzed, and the patients were divided into groups according to the course over the following weeks and the results of prescribed treatment: group 1 (effective treatment), group 2 (ineffective treatment), and group 3 (treatment dropout).

Based on the prescription history, corroborated with drug retrieval and the acquisition costs (FARMASYS® software), the cost of antiretroviral medication was calculated for each of the 108 patients receiving highly active antiretroviral therapy (HAART). The statistical analysis was carried out with the SPSS version 11.5 statistical package, and considering statistical significance for $p<0.05$.

Results: The cost generated by the 108 patients included during the study period who received HAART was $232,366 €$, with an average of $2,151 €$ per patient and 19,363 € per day of consultation. The distribution of patients and of the costs generated by the above defined groups was as follows: group 1: 79 patients (80.6\%); group 2: 11 patients (10.6\%); group 3: 18 patients $(8.8 \%)$. In the group 1 patients, $15.2 \%$ of the antiretroviral expenditure corresponded to patients who had started therapy with a CD4+ count of $>350 \mathrm{cells} / \mathrm{ml}$, while $45 \%$ had $>350$ cells $/ \mathrm{ml}$ at the present time, and $25.9 \%$ had $>500 \mathrm{cells} / \mathrm{ml}$. Previous medication dropout was identified as a predictor of new dropouts and treatment failures.

Comment: While the cost generated by antiretroviral medication is high, $19.4 \%$ is administered to patients who do not benefit from such treatment due to a lack of efficacy or dropouts, and in another 40-60\% of cases the need for such treatment could be subject to consideration in view of the good immune status of the patients.

KEY WORDS: Antiretroviral therapy. Drugs cost.

Tornero Estébanez, C, Cuenca Soria A, Nolasco Bonmatí A, Soler Company E, Rull Segura S. Gasto farmacéutico en medicación antirretroviral y posibilidades de optimización. An Med Interna (Madrid) 2005; 22: 575-578.

Trabajo fiananciado por AISSA (Asociación para la Investigacion Sanitaria de la Safor).

Trabajo aceptado: 29 de julio de 2005

Correspondencia: Carlos Tornero. Servicio de Medicina Interna. Hospital Francisco de Borja. Paseo Germanias, 71.46700 Gandía. Valencia. e-mail: tornero_cas@gua.es 


\section{INTRODUCCIÓN}

Las combinaciones de fármacos antirretrovirales han demostrado su utilidad en la mejoría de la supervivencia de los pacientes con infección por VIH $(1,2)$. Las peculiaridades socioculturales de estos pacientes hacen que no todos los pacientes a los que se les prescribe el tratamiento antirretroviral de gran actividad (TARGA) se beneficien de él. Son frecuentes los abandonos y la ineficacia por resistencias o falta de adherencia, lo que puede suponer un gasto económico poco útil. Por otra parte la reconstitución inmunológica y la adopción de recomendaciones más conservadoras para iniciar el tratamiento, ha generado un grupo de pacientes donde podría no ser necesario.

Con la experiencia previa de un estudio retrospectivo (3), nos planteamos cuantificar mediante un protocolo prospectivo, como se distribuye el gasto farmacéutico en medicación antirretroviral en un hospital comarcal; según el resultado del tratamiento y la relación con ciertas variables del paciente se analizarán las posibilidades de mejorar su eficiencia.

\section{MATERIAL Y MÉTODOS}

Se incluyeron todos los pacientes con infección por VIH atendidos durante 12 semanas consecutivas (septiembre a noviembre del 2004) en una consulta de Medicina Interna semanal polarizada al seguimiento de esta infección. En aquellos en los que se decidió iniciar, cambiar o mantener el TARGA se recogieron las siguientes variables: edad, sexo, grupo de riesgo, tratamiento con metadona, tiempo de tratamiento actual, prescripción realizada y antecedentes de abandonos previos, entendiendo como tal cuando se tenía la constancia que un paciente había interrumpido previamente la medicación por iniciativa propia durante más de 15 días.

Entre las 12 y las 16 semanas siguientes se realizó un control clínico y de eficacia virológica, clasificando los pacientes según los resultados en los siguientes grupos:

-Grupo 1 o de tratamiento eficaz: pacientes que mantienen carga viral por debajo de 200 copias/ml como efecto del tratamiento antirretroviral.

-Grupo 2 o de tratamiento no eficaz: aquellos que pese a acudir a las revisiones y recoger la medicación de forma regular, presentan viremias para VIH por encima de las 1.000 copias/ml. En los pacientes con viremias entre 200 y 1.000 se repitió la determinación para clasificarlos en alguno de los grupos anteriores.

-Grupo 3 o abandonos de tratamiento: pacientes que no acuden a la consulta programada ni en los 15 días siguientes.

Aquellos en los que se inició o modificó el tratamiento se realizó un control clínico de tolerancia y adherencia a los 15 días, citándose para el control virológico a las 6-8 semanas y clasificándose en ese momento en los mismos grupos ya descritos; se consideró tratamiento eficaz y perteneciente al grupo 1 cuando el descenso de viremia era acorde con eficacia del tratamiento para el tiempo transcurrido.

En los pacientes que no acudieron a las consultas se intentó contactar telefónicamente para indagar sobre la causa de su falta de asistencia. Sólo se incluyó a cada paciente en una sola ocasión, aunque tras un abandono, el paciente volviera a la consulta y se reiniciase el seguimiento.
La dispensación de la terapia antirretroviral fue realizada por el servicio de farmacia en cantidad suficiente hasta la siguiente revisión clínica pero nunca para más de 30 días, debiendo los pacientes volver a recoger la medicación mensualmente.

Con la historia de prescripción corroborada con la recogida de medicación y los costos de adquisición (programa informático FARMASYS ${ }^{\circledR}$ ), se calculó el gasto en medicación antirretroviral generado en cada paciente. Los costos de adquisición coinciden con los publicados recientemente (4) salvo para el caso de la zidovudina genérica cuyo importe mensual a la dosis de $300 \mathrm{mg} / 12 \mathrm{~h}$ es de $30 €$.

La recogida de datos clínicos se realizó mediante el programa HandBase 3.0 para Palm y el análisis estadístico mediante SPSS 11,5. Para el análisis de asociación entre las variables cualitativas se utilizó el test Chi cuadrado, mientras que las diferencias de medias entre las variables cuantitativas se calcularon con la prueba de t Student. Para evaluar los efectos ajustados fueron construidos modelos de regresión logística. El nivel de significación estadística se estableció en $\mathrm{p}<0,05$.

\section{RESULTADOS}

Durante el periodo de inclusión del estudio, se atendieron 128 pacientes. En 20 de ellos no se pauto TARGA porque las condiciones inmunológicas, de toxicomanía activa ( 2 casos) o de patología psiquiátrica grave (1 caso) no lo hacían recomendable.

Durante el año 2004, 218 pacientes adultos distintos con infección por VIH recogieron medicación en el Hospital de Gandia, al menos una vez, no incluidos en ensayos clínicos ni como pautas de prevención postexposición. El consumo anual de la medicación antirretroviral fue de 1.193.932 €, aproximadamente el $21 \%$ de la cantidad total destinada a medicación por el servicio de Farmacia y un aumento de un $7 \%$ respecto al año anterior.

El gasto total generado en los 108 pacientes incluidos en el estudio que recibían TARGA fue de $232.366 €$, lo que supone una media de $2151 €(\mathrm{DE} 709,7)$ por paciente y $19363 €$ por día de consulta.

La distribución de los pacientes y del gasto generado por los grupos antes definidos es la siguiente (Fig. 1):

-Grupo 1: tratamiento eficaz: 79 pacientes, $18.7293 €$ $(80,6 \%)$.

-Grupo 2: Tratamiento no eficaz: 11 pacientes $24.520 €$ $(10,6 \%)$.

-Grupo 3: Abandonos del tratamiento: 18 pacientes: $20.553 €(8,8 \%)$.

Para conocer las causas del abandono del tratamiento (grupo 3), se intentó contactar telefónicamente, consiguiéndose en 13 de los sujetos; sólo uno refirió que por motivos laborales había recogido la medicación en otra ciudad, pero que el seguimiento seguiría siendo en nuestro centro por lo que se le dieron nuevas citas a las que no acudió. Los restantes argumentaron motivos diversos para justificar su falta de asistencia; muchos de ellos reiniciaron el seguimiento, y algunos ya han abandonado de nuevo. El análisis de los factores que se asociaban a las perdidas de seguimiento (grupo 3) se realizó con el test de la chi cuadrado para las variables cualitativas y t 


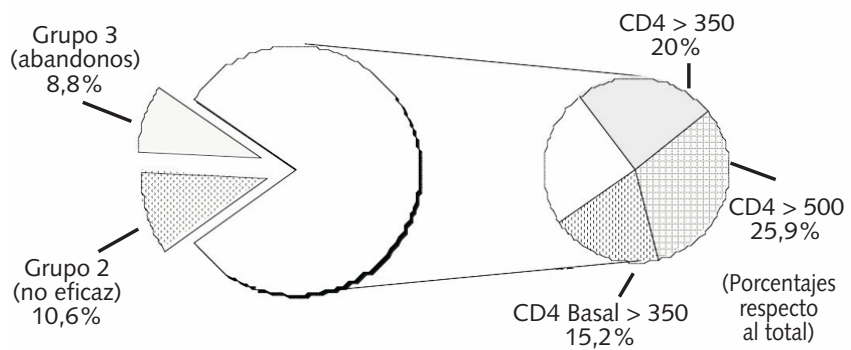

Fig. 1. Distribución del gasto en los diferentes grupos.

student para las cuantitativas. No se encontraron diferencias significativas por edad, sexo, ni grupos de riesgo. El antecedente de tratamiento con metadona $(\mathrm{p}=0,047)$ y sobretodo de abandonos anteriores ( $\mathrm{p}<0,01$, RR 23,8, IC 95\% 3,2-172,4) resultaron predictoras de nuevas pérdidas de seguimiento. Aplicando un modelo de regresión logística para evaluar el efecto ajustado de cada variable, sólo el antecedente de abandonos previos mantuvo la significación estadística.

De la misma manera el antecedente de abandonos previos fue el único factor que se asoció de manera significativa con un resultado de tratamiento no eficaz (grupo 2) frente a un tratamiento eficaz. ( $\mathrm{p}<0,01$. RR 8,49 IC95\% 1,35-55,25).

En los pacientes donde el tratamiento fue eficaz (grupo 1) se analizó la distribución según la cifra de linfocitos CD4 en el momento que iniciaron la TARGA y en el momento actual: en 14 casos el tratamiento se había iniciado con CD4 > 350 $\mathrm{cs} / \mathrm{ml}$ lo que representaba $35.340 €(15,2 \%$ del gasto total). En los 65 restantes la distribución según la cifra de CD4 actual era la siguiente: 25 estaban con un recuento de células mayor de 500/ml, lo que representaba el 25,9\% del gasto farmacológico, y 19 más con cifras por encima de $350 \mathrm{cs} / \mathrm{ml}$ $(20,9 \%)$ (Fig. 1).

\section{DISCUSIÓN}

La introducción de las combinaciones terapéuticas de antirretrovirales ha mejorado la supervivencia de los pacientes con infección por VIH. Se ha descrito incluso que, pese al elevado gasto farmacológico generado, se reducen los costos globales generados por estos pacientes $(1,2)$. La incuestionable utilidad de estos fármacos no debe obviar la necesidad de optimizar y racionalizar el coste generado, identificando escenarios y factores que permitan su uso más eficiente. Los resultados presentados aquí permiten apuntar líneas de trabajo en este sentido.

La primera de las consideraciones debería hacerse respecto a los pacientes que, tras recoger la medicación, no acuden a controles analíticos ni a las consultas. Generan un gasto económico poco útil, además de un elevado riesgo de generar resistencias a la medicación antirretroviral. En nuestra serie suponen 8,8\% del costo de la medicación administrada (18 pacientes) además de asociarse a fracasos posteriores. Aunque no puede descartarse que algún paciente pasara a ser seguido en otro centro, no lo objetivamos en aquellos con los que se consiguió contactar telefónicamente. Los antecedentes pre- vios de abandonos fue el factor que se comportó como predictor en estas pérdidas de seguimiento pues 17 de los 18, ya habían interrumpido antes otras pautas terapéuticas. Además en el 83,4\% el abandono se dio en los primeros 6 meses del tratamiento, incluyendo los 8 casos en los que se produjo tras la primera dispensación. Un gran esfuerzo de refuerzo de la adherencia y la administración de la primera medicación para sólo 15 días en los pacientes que inician un tratamiento nuevo, permitiría detectar precozmente un porcentaje elevado de las pérdidas de seguimiento y reducirían sus costos. Si la presión asistencial lo permitiera, el control clínico más estrecho y la administración de medicación para periodos más cortos de tiempo, al menos en los primeros meses, y sobretodo en los pacientes con historia previa de abandonos, mejorarían la adherencia y/o reduciría este gasto farmacológico poco útil.

Los pacientes que, pese a acudir regularmente a la recogida de la medicación y a las revisiones clínicas, no alcanzan los objetivos terapéuticos de suprimir la viremia, son, en muchos casos, resultado de adherencias inadecuadas, resistencias y/o la sucesión de ambas circunstancias. En nuestra serie alcanzan hasta el 10,6\% del gasto en mediación antirretroviral. Estrategias para mejorar la adherencia, incluso complejas, pueden resultar costo-efectivas en estos pacientes no cumplidores, especialmente en fases evolucionadas de la enfermedad (5). Los antecedentes de abandonos previos son también en nuestra serie predictores de este riesgo de fracaso.

El volumen más importante y por tanto donde las posibilidades de ahorro serían mayores, corresponde a pacientes que mantienen viremias indetectables. En ellos se podría plantear la utilización de pautas de tratamiento más económicas y la interrupción del mismo en determinados escenarios.

La elección entre las diferentes combinaciones de TARGA posibles es un proceso complejo donde las consideraciones económicas deben, probablemente, también considerarse, pues pueden existir diferencias en el coste de hasta un $40 \%$ entre las consideradas pautas preferentes (4). La posibilidad de prescripción de medicamentos genéricos está limitada a la ziduvidina e implicaría, en la mayoría de las ocasiones, sustituir las co-formulaciones que la contienen (Combivir ${ }^{\circledR}$ y Trizivir $^{\circledR}$ ) y por tanto aumentar el número de comprimidos. En el caso de Combivir ${ }^{\circledast}$ el pasar de 2 comprimidos a los 3 que suman la zidovudina genérica y la lamivunida, supondría una reducción del $60 \%$ del costo de adquisición, en un medicamento que de por sí solo representa el 18\% del costo económico en TARGA en nuestro hospital. La utilización real en nuestro centro de zidovudina genérica es anecdótica $(0,1 \%)$.

La posibilidad de interrumpir el tratamiento antirretroviral tras la reconstitución inmunológica requiere múltiples consideraciones sobre la posibilidad de progresión clínica, generación de resistencias, reducción de toxicidad y disminución del gasto. Aunque no están exentas de riesgos (6), existen experiencias que apuntan a la utilidad de estas estrategias (7-10) sobretodo cuando el tratamiento se inició con cifras de CD4 elevadas. En nuestros pacientes podría aplicarse a un $15 \%$ del gasto generado en pacientes que iniciaron tratamiento con un recuento de linfocitos CD4 elevado, y a otro 45 o $25 \%$ correspondiente a pacientes que han alcanzado gracias a la TARGA cifras por encima de 350 y 500 células $/ \mathrm{ml}$ respectivamente. Aunque supondría un gran ahorro económico, no es una práctica recomendada de forma rutinaria salvo en el contexto de estudios clínicos (4). Los próximos años nos permitirán sopesar los beneficios económicos y de reducción de toxicidad con 
los riesgos apuntados y establecer estrategias de tratamiento que pueden modificar la carga económica de la TARGA.

En conclusión el gasto en medicación antirretroviral es elevado pero se pueden plantear estrategias para reducirlo de forma considerable. Los pacientes con abandonos previos son los de mayor riesgo de nuevos abandonos o fracasos y por tanto un grupo donde podrían centrarse los esfuer-

\section{Bibliografía}

1. Bozzete SA, Joyce G, McCaffrey DF, Leibowitz AA, Morton SC, Berry $\mathrm{SH}$, et al. Expenditures for the care of HIV-infected patients in the era of highly active antiretroviral therapy. N Engl J Med 2001; 344: 817-23

2. Yazdanpanah Y. Costs associated with combination antiretroviral therapy in HIV-infected patients. J Antimicrobiol Chemother 2004; 53: 558-561.

3. Tornero C, Cuenca A, Santamaría A, Gil E, Soler E, Rull S. Distribución del gasto farmacéutico en medicación antirretroviral. An Med Interna (Madrid) 2004; 21: 269- 271.

4. Iribarrena, JA, Labarga P, Rubio R, Berenguer J, Miró JM, Antela A, et al. Recomendaciones de GESIDA/Plan Nacional sobre el Sida respecto al tratamiento antirretroviral en pacientes adultos infectados por el VIH (octubre 2004) Enferm Infecc Microbiol Clin 2004; 22: 564-642.

5. Goldie SJ, Paltiel AD, Weinstein MC, Losina E, Seage GR 3rd, Kimmel $\mathrm{AD}$, et al. Projecting the cost-effectiveness of adherence interventions in persons with human immunodeficiency virus infection. Am J Med 2003; 115: 632-41. zos de mejorar eficacia y eficiencia. La opción de la retirada temporal de la medicación ofrece también grandes posibilidades, pero su aplicación clínica está por definir. En cualquier caso ninguna estrategia teórica de ahorro de costos será útil sin la dotación de medios y la implicación del médico prescriptor mediante políticas de motivación que deben articularse.

6. Schweighardt B, Ortiz GM, Grant RM, et al. Emergence of drug-resistant HIV-1 variants in patients undergoing structured treatment interruptions. AIDS 2002; 16: 2342-44.

7. Tarwater PM, Parish M, Gallant JE. Prolonged treatment interruption after immunologic response to highly active antiretroviral therapy. Clin Infect Dis 2003; 37: 1541-48.

8. Fagard C, Oxenius A, Guntharf $\mathrm{H}$, et al. A prospective trial of structured treatment interruptions in human immunodeficiency virus infection. Arch Intern Med 2003; 163: 1220-1226.

9. Skiest DJ, Morrow MD, Allen B, McKinsey J, Crosby C, Foster B, et al. It is safe to stop antiretroviral therapy in patients wit preantiretroviral CD4 cell counts > 250 cells/uL. J Acquir Immune Defic Syndr 2004; 37: 3: 1351-1357.

10. Maggiolo F, Ripamonti D, Gregis G, Quinzan G, Callegaro A, Suter F. Effect of prolonged discontinuation of successful antiretroviral therapy on CD4 cells: a controlled, prospective trial. AIDS 2004; 18 : 439-446. 